\title{
Image Segmentation Technique Using SVM Classifier for Detection of Medical Disorders
}

\author{
Ummadi Janardhan Reddy ${ }^{*}$, Pandluri Dhanalakshmi ${ }^{2}$, Pallela Dileep Kumar Reddy ${ }^{3}$ \\ ${ }^{1}$ Vignan's Foundation for Science Technology \& Research (Deemed to be University), Vadlamudi, Guntur, India \\ ${ }^{2}$ Department of CSSE, Sree Vidyanikethan Engineering College, A. Rangampet-517102, Tirupathi, India \\ ${ }^{3}$ CSE Department, SV College of Engineering, Karakambadi Road-517507, India
}

Corresponding Author Email: ummadi.janardhan@gmail.com

https://doi.org/10.18280/isi.240207

Received: 5 January 2019

Accepted: 23 March 2019

\section{Keywords:}

MRI image, SVM, brain tumor, correlation, edge detection, image segmentation

\begin{abstract}
A support vector machine (SVM) classifier is being proposed for characterization of cerebrum tissues in attractive reverberation photographs (MRI). A wavelet based absolutely surface highlights set is determined. The most helpful surface capacities are extricated from standard just as tumor districts through the utilization of spatial dark dimension reliance strategy. The proposed strategy settles the enormous inconvenience of class procedures. These most proficient capacities are then used to classifications the cerebrum tissues into favorable and threatening tumor. The general execution of the arrangement of principles is assessed completely dependent on a succession of mind tumor pictures.
\end{abstract}

\section{INTRODUCTION}

The principle of adopted task is to acknowledge the growth from a specific imaging scan of brain image exploitation using digital image process techniques and reckon the world of the growth. The high resolution pictures are accustomed to examine human brain development and see abnormalities [1].

Mixture Tumors are sleek, spherical lesions of endodermis origin, generally situated at the opening of H. H. Munro within the anterior side of the ventricle. These Tumors usually occur in adults and account for about one hundred and twenty fifth of all intracranial tumors. On non-enhanced computerized tomography (CT), the bulk of mixture Tumors are hyper dense to brain tissue [2]. A skinny rim of improvement is visible once endogenous distinction administration. Owing to the super molecule nature of its contents, a mixture growth is usually hyper dense on T1-weightedresonance (MRI) pictures and hypo intense on T2-weighted adult male [3].

Typically, mixture Tumors are actinically silent and are found incidentally once patients are imaged for different reasons [4]. Whereas some little mixture Tumors could never cause any symptoms, those who have grownup to be sufficiently large in size will block the natural flow of spinal fluid (CSF) and cause hydrocephaly and its associated symptoms like headaches, weakness of the limbs [5], and loss of consciousness. These symptoms are also intermittent owing to transient episodes of hydrocephaly that are caused by mobile Tumors that often block the flow of CSF [6]. Different symptoms could be vomit, nausea, blurred or visual disorder, downward deviation of the eyes, issues with balance and coordination, incontinence, drowsiness, altered mental standing, coma, or different changes in temperament or noses together with state of mind [7]. Mixture Tumors are generally diagnosed through clinical medical specialty analysis and by exploitation of brain imaging techniques like computerized tomography or resonance imaging (MRI) [8]. These imaging modalities will usually demonstrate Tumor's structure that's basically blocking the flow of CSF likewise as any associated hydrocephaly. Magnetic Resonance Imaging (MRI) is a complicated medical imaging technique accustomed to turn out high resolution pictures of the elements contained within the shape. Imaging is commonly used once treating mixture growth [9].

\section{LITERATURE SURVEY}

Many a times it becomes difficult to distinguish the benign tumor from the malignant ones. With the resource of picture processing methods which include mathematical morphology that actually define the threshold of the snap shots, it turns into less complicated to pick out the scale, form and different characteristics of pathological mobile images [10].

The rims of a photograph continually consist of various inherent information (along with step individual, direction, form, and so on.), which are large attributes for extracting features in image recognition. In most of the cases, pixels alongside and the edge changes regularly, while those being perpendicular the brink generally have sharp modifications [11].

Normally, part extraction arithmetic's is to stumble on mathematical operators of the pixels which can be being coincident with the capabilities of the brink. Canny derived analytically, the most excellent step part operators and confirmed that the primary spinoff Gaussian filter out is a superb approximation for all such operators [12]. Statistical technique is an opportunity to gradient techniques. Figuring out whether or not a pixel wishes to be categorized as part primarily based on depth values of the neighborhood being the simple concept [13].

In evaluation to the differential procedures, much less attention is being paid to statistical methods. But the ones methods have already been approached through some authors [14]. In different words we can say that an aspect is not a bodily entity, similar to a shadow. This is where the wall starts and the photo ends. It's miles here in which the vertical and the horizontal surfaces of an item meet [15]. It has no width because it is in between a bright window and the darken of the 
proper. Part detection basically incorporates the following parts: -

The use of side operators the edge factor set extracted.

A few aspect points in the edge point set are removed.

Then, the received facet points are connected to be a line. Generally we use following operators for side detection e.g., canny, log, binary morphology and differential operator.

In [16] it is shown that erosion and dilation operations have higher effect on photograph area. This is decided through appearing the difference among processed photo and original picture. It's also shown that erosion and dilation operations are worse for noise filtering. Rather, it's far located that opening and closing operations are higher for filtering than erosion and dilation operations. In brain MRI, the correct place of tumor can be proved to be very crucial inside the early detection of pathological tissues [17]. This detection additionally allows in minimizing the harm to healthful tissues, which may be because of any remedy technique, like radio surgical treatment etc.

The brain fluid attenuated inversion recovery (aptitude) MRI offers a few treasured methods to perform both pre and publish-surgical opinions [18]. Maximum of the mind segmentation work done till now become based totally on $\mathrm{t}$, t2 \& pd weighted images where we had used fluid attenuated inversion recuperation (flair) photographs which might be widely widespread for brain diagnosis. Flair sequences are people who produce heavily cerebrospinal fluid (CSF) and t2 weighted nulled MRI snap shots. With this approach, subtle lesions near the CSF, stand out against a returned floor of attenuated CSF fluids such that cerebrospinal fluid (CSF) seems darkish and maximum lesions, tumors and edematous tissues appear quite vivid, supplying stepped forward distinction among hyper intense lesions and adjacent fluid stuffed cavities via suppressing the fluid sign [19].

The brain fluid attenuated inversion recovery (aptitude) MRI offers a few treasured methods to perform both pre and publish-surgical opinions. Maximum of the mind segmentation work done till now become based totally on t1, t2 \& pd weighted images where we had used fluid attenuated inversion recuperation (flair) photographs which might be widely widespread for brain diagnosis. Flair sequences are people who produce heavily cerebrospinal fluid (CSF) and t2 weighted nulled MRI snap shots. With this approach, subtle lesions near the CSF, stand out against a returned floor of attenuated CSF fluids such that cerebrospinal fluid (CSF) seems darkish and maximum lesions, tumors and edematous tissues appear quite vivid, supplying stepped forward distinction among hyper intense lesions and adjacent fluid stuffed cavities via suppressing the fluid sign [20].

In all of the above referred to paintings, both case research has been done or tumors alone are detected using aptitude photos but in all previous work simplest flair photographs are used to detect tumor, wm, gm and CSF using combined residences of wavelet and statistical parameters of function vector. Many authors used residences of wavelet transform. Our proposed method is based on image segmentation and clustering. Proposed segmentation method off course better than existing segmentation methods likes Particle swarm optimization (PSO), Fuzzy C-Means SVM [21] and many more.

Function $f(e)$ gives better result than any one of image filtering. here ef is an enhancement factor that will be decided based on image mean intensity [22].
In r. Rajeswari et al. [9] proposed a spectral leakage has the effect of the frequency evaluation of finite-duration segments or finite-period alerts of countless signals. In brain the tumor itself, comprising a necrotic (lifeless) part and an active component, the edema or swelling in the near-by brain, as all tumor do now not have a clear boundary between energetic and necrotic element, there's an urge to define a clean boundary among brain tissues and edema. P.narendran, v.k. Narendira kumar, k. Somasundaram [10] proposed a brand new approach for segmentation of pathological brain systems. This approach combines earlier facts for segmentation of systems and photograph records (region and aspect) [23]. The automated mind tumor segmentation method that has been developed includes important components: pre-processing and segmentation [24]. The 2 specific modalities of MRI images which might be inputs of this system are: ce-t $1 \mathrm{w}$ and aptitude that we consider are sufficient for mind tumor segmentation [25].

\section{PROPOSED METHODOLOGY}

Let an image $i$ which is an MRI image taken from standard database source. Initially an Image $i$ get enhance so that any disturbance or noise get reduced. $i i=1 \quad 3$

$$
\begin{aligned}
& \text { if } \mathbf{m i}>=0 \& \& \mathbf{m i}<0.3 \text { ef }=\mathbf{0 . 6} \\
& \text { end } \\
& \text { if } m i>=0.3 \& \& m i<0.6 \text { ef }=0.7 \\
& \text { end } \\
& \text { if } m i>=0.6 \& \& m i<0.9 \text { ef }=0.8 \\
& \text { end } \\
& \text { if } m i>=0.9 \& \& m i<1.0 \text { ef }=0.9 \text { end }
\end{aligned}
$$

Once an image gets enhance, it goes for ROI region selection. We are considering that background region of MRI image is always black that differentiate ROI region from it. We are scanning MRI image from left to right and top to bottom Raster Scanning) way and counting for back pixels. If there are continuous ten black pixels are occurred, then algorithm considered these pixels as a background pixel else not.

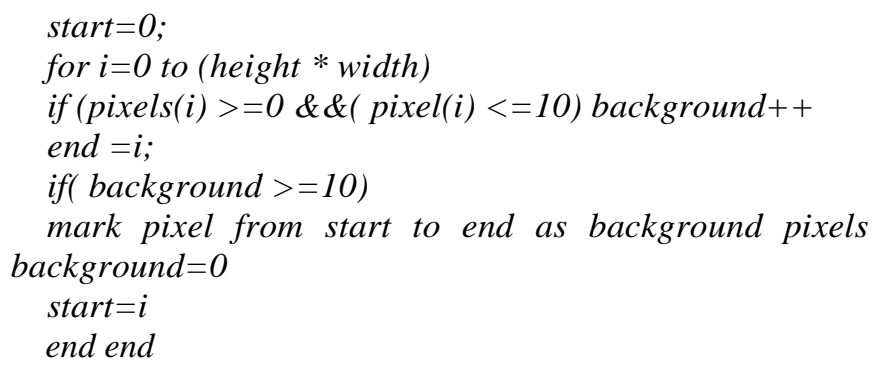

Selecting ROI of an image is a vital task because accuracy depends on it. Wrong selection may lead to inaccurate result.

Selecting ROI of an image is a vital task because accuracy depends on it. Wrong selection may lead to inaccurate result.

In proposed methodology, infected region highlighted with intensity of a pixel. Generally tumor region is identified with its whiteness color because of its nature, but range of white color varies pixel value from 225 to 255 in an image intensity region, which creates confusion. Our proposed methodology, we set threshold value that perfectly classify infected region as ppiippeepp $\geq 225$ aaaaaa $\leq 255$. 


\section{EXPERIMENT RESULTS}

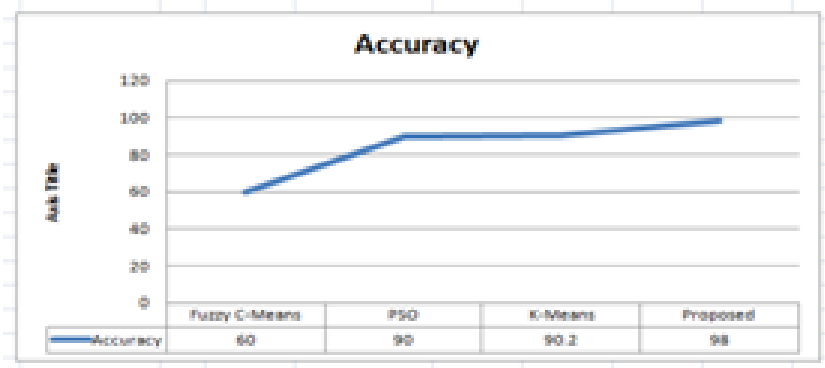

Figure 1. Accuracy

\section{ANALYSIS OF PROBLEMS}

Scientific photograph segmentation is a way employed in areas of diagnosis and detection of lesions, tumors or other abnormalities. It is also helpful in surgical evaluation and submit-surgical evaluation of the affected person. It has already been used for tumor reputation and for figuring out tumor limitations. Photograph segmentation techniques are usually used in scientific area for detecting diseases like nerves harm, blood vessels extraction and tumor detection in human frame systems. Magnetic resonance imaging (M RI) is a technique of non-invasive nature for scientific imaging that uses the pulses of radio waves and magnetic area. It offers better visualization of soft tissues found in human body.

The erosion and dilation operations deliver better effect for photograph part with the aid of acting the difference between processed picture and authentic photo, but they provide bad result for noise filtering. Hence in these paintings we examine erosion and dilation problem with the assist of merging this morphological output in which colloid tumor receives detected sharply. The simple reason of the operations is to focus on that part of the image which has the tumor i.e. the part of the photograph having greater depth and greater location than that distinct inside the strel command. The simple instructions used in this step are strel, imerode and imdilate. Imerode is used to erode a picture and Imdilate is used to dilate a photo. On merging these morphological outputs with grayscale photograph we get resultant output in which colloid tumor gets detected sharply.

The inherent data about any picture (which includes course, step individual, shapes and so forth.) Is contained in the edges. Those are considerable attributes for characteristic extraction in photograph reputation. A sluggish exchange is discovered in pixels along an edge, while those perpendicular to the direction of the threshold exhibit sharp changes. In preferred, mathematics for facet extraction is to test for the twist of fate among mathematical operators of the pixels and capabilities of the rims.

It is depicted form Figure 1 the proposed methodology promising more accurate results than existing clustering techniques. Accuracy of proposed methodology is $98 \%$ which is far better than other existing techniques (Fuzzy C-means, PSO and $\mathrm{k}$ means).

The time comparison of various methods has been depicted in Figure 2. We also carried out simulation on over $100 \mathrm{MR}$ Images under standard LAB environment conditions and comparative results has been shown in Figure 3.

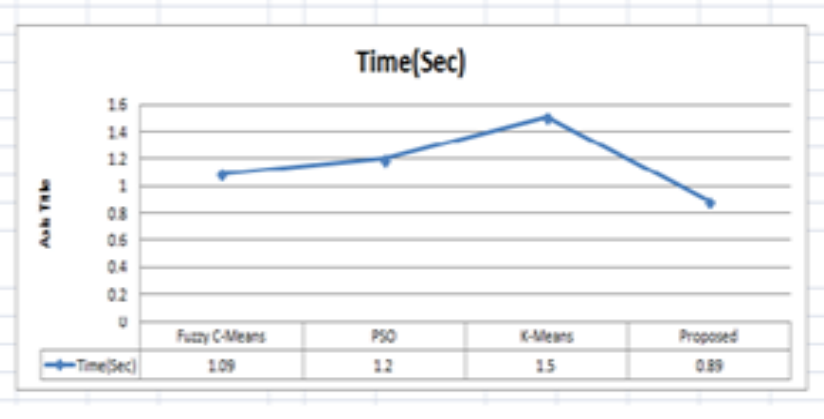

Figure 2. Time comparison

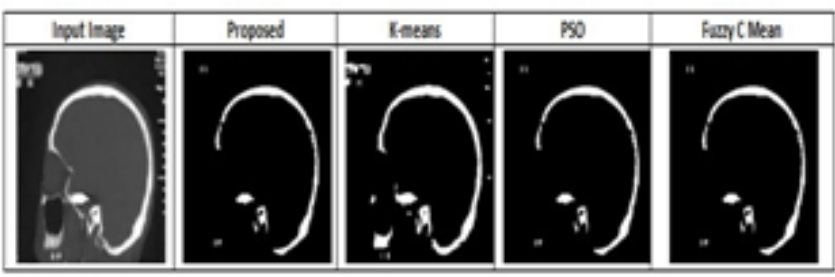

Figure 3. Comparative study

\section{APPLICATION}

Proposed algorithm has major advantage of identifying different Tumor sizes, intensities and locations with a good accuracy. Tumor detection in MRI brain image is useful in medical science. Also, it is used for study of anatomical structure.

Identify Region of Interest i.e. locate tumor, lesion and other abnormalities.

Measurement of tissue volume to measure growth of tumor (also decrease in size of tumor with treatment).

Help in planning the treatment prior to radiation therapy and in radiation dose calculation.

\section{DISCUSSION \& CONCLUSION}

The crux of this article is support vector machine (SVM) classifier to develop medical decision system using edge detection \& matrix correlation. The proposed approach gives very promising results in classifying the healthy and pathogen affected brain. The system is beneficial in assisting the physician to make the final decision. This is efficient for classification of the human brain as normal or abnormal (benign and malignant Tumor) with high rate of sensitivity and accuracy. The technique is accurate, robust, easy to operate, non-invasive and cheap which is advantageous.

\section{REFERENCES}

[1] Chang, R.F., Wu, W.J., Moon, W.K., Chou, Y.H., Chen, D.R. (2003). Support vector machines for diagnosis of breast tumors on US images. Academic Radiology, 10(2): 189-197. https://doi.org/10.1016/S1076-6332(03)800442

[2] Chang, R.F., Wu, W.J., Moon, W.K., Chou, Y.H., Chen, D.R. (2003). Improvement in breast tumor discrimination by support vector machines and speckle- 
emphasis texture analysis. Ultrasound in Medicine and Biology, 29(5): 679-686. https://doi.org/10.1016/s03015629(02)00788-3

[3] Antonie, L. (2008). Automated Segmentation and Classification of Brain Magnetic Resonance Imaging, C615 Project.

[4] Chaplot, S., Patnaik, L.M., Jagannathan, N.R. (2006). Classification of magnetic resonan ce brain images using wav elets as input to support vector machine and neural network. Biomedical Signal Processing and Control, 1(1): 86-92. https://doi.org/10.1016/j.bspc.2006.05.002

[5] Gering, D.T., Eric, W., Grimson, L., Kikins, R. (2002). Recognizing deviations from normalcy for brain tumor segmentation. Lecture Notes in Computer Science, 2488: 388-395. https://doi.org/10.1007/3-540-45786-0_48

[6] Schad, L.R., Bluml, S., Zuna, I. (1993). MR tissue characterization of intracranial tumors by means of texture analysis. Magnetic Resonance Imaging, 11(6): 889-896. https://doi.org/10.1016/0730-725x(93)90206-s

[7] Freeborough, P.A., Fox, N.C. (1998). MR image texture analysis applied to the diagnosis and tracking of Alzheimer's disease. IEEE Transactions on Medical Imaging, 17(3): 475-479. https://doi.org/10.1109/42.712137

[8] Dunn, C., Higgins, W.E. (1995). Optimal Gabor filters for texture segmentation. IEEE Transactions on Image Processing, 4(7): 947-964. https://doi.org/10.1109/83.392336

[9] Chang, T., Kuo, C. (1993). Texture Analysis and classification with tree structured wavelet transform. IEEE Transactions on Image Processing, 2(4): 429-441. https://doi.org/10.1109/83.242353

[10] Siedlecki, W., Sklanky, J. (1989). A note on genetic algorithms for large- scale feature selection. Pattern Recognition Letters, 10(5): 335-347. https://doi.org/10.1016/0167-8655(89)90037-8

[11] Haralick, R.M., Shanmugam, K., Dinstein, I. (1973). Textural features for image classification. IEEE Trans On Systems Man and Cybernetics, 3(6): 610-621. https://doi.org/10.1109/TSMC.1973.4309314

[12] Soh, L., Tsatsoulis, C. (1999). Texture analysis of SAR sea ice imagery using gray level Co-occurrence $M$ atrices. IEEE Transactions on Geoscience and Remote Sensing, 37(2): 780-793. https://doi.org/10.1109/36.752194

[13] Clausi, D.A. (2002). An analysis of co-occurrence texture statistics as a function of grey level quantization. Canadian Journal of Remote Sensing, 28(1): 45-62. https://doi.org/10.5589/m02-004

[14] Kharrat, A., Messaoud, B.M., Benamrane, N., Abid, M. (2009). Detection of brain tumor in medical images.
IEEE 3rd International Conference on Signals, Circuits \& Systems, 6. https://doi.org/10.1109/ICSCS.2009.5412577

[15] Goldberg, D.E. (1989). Genetic Algorithms in search, optimization and machine learning. Boston, MA, USA, Addison-Wesley Longman.

[16] Vapnik, V.N. (1982). Estimation of dependences based on Emp irical data. Secaucus. NJ, USA, Springer-Verlag New York. https://doi.org/10.1007/0-387-34239-7

[17] Guyon, I., Weston, J., Barnhill, S., Vapnik, V. (2002). Gene selection for cancer classification using support vector machines. Machine Learning, 46(1-3): 389-422. https://doi.org/10.1023/a:1012487302797

[18] Zhang, J., Liu, Y. (2004). Cervical can cer detection using SVM based feature screening. Proc of the 7 th Medical Image Computing and ComputerAssistedIntervention, $\quad 2$ : 873-880. https://doi.org/10.1007/978-3-540-30136-3_106

[19] Zhang, K., Cao, H.X., Yan, H. (2006). Application of support vector machines on network abnormal intrusion detection. Application Research of Computers, 5: 98-100. https://doi.org/ 10.1360/crad20061223

[20] Kim, D., Park, J. (2003). Network-based intrusion detection with support vector machines. Lecture Notes in Computer Science, 2662: 747-756. https://doi.org/10.1007/978-3-540-45235-5_73

[21] Ricci, P.E., Dungan, D.H. (2001). Imaging of low- and intermediate- grade gliomas. Seminars in Radiation Oncology, $11(2)$ : 103-112. https://doi.org/10.1053/srao.2001.21420

[22] Armstrong, T.S., Cohen, M.Z., Weinbrg, J., Gilbert, M.R. (2004). Imaging techniques in neuro oncology. In Seminars in Oncology Nursing, 20(4): 231-239. https://doi.org/10.1016/j.soncn.2004.07.003

[23] Shubhangi, D.C., Hiremath, P.S. (2009). Support vector machine (SVM) classifier for brain tumor detection. International Conference on Advances in Computing. Communication and Control, 444-448. https://doi.org/10.1145/1523103.1523191

[24] Reddick, W.E., Glass, J.O., Cook, E.N., Elkin, T.D., Deaton, R.J. (1997). Automated segmentation and classification of multispectral magnetic resonance images of brain using artificial neural networks. IEEE Transactions on Medical Imaging, 16(6): 911-918. https://doi.org/10.1109/42.650887

[25] Clark, M.C., Hall, L.O., Goldgof, D.B., Velthuizen, R., Murtagh, F.R., Silbiger, M.S. (1998). Automatic tumor segmentation using knowledge based techniques. IEEE Transactions on Medical Imaging, 17(2): 187-192. https://doi.org/ 10.1109/42.700731 